\title{
Labrador Sea sub-surface density as a precursor of multi-decadal variability in the North Atlantic: a multi-model study
}

Pablo Ortega ${ }^{1,2}$, Jon Robson ${ }^{1}$, Matthew Menary ${ }^{3}$, Rowan Sutton ${ }^{1}$, Adam Blaker $^{4}$, Agathe Germe $^{4}$, Jöel Hirschi ${ }^{4}$, Bablu Sinha ${ }^{4}$, Leon Hermanson ${ }^{5}$, and Stephen Yeager ${ }^{6}$

${ }^{1}$ NCAS, University of Reading, Reading, UK

${ }^{2}$ Barcelona Supercomputing Center, Barcelona, Spain

${ }^{3}$ LOCEAN, Sorbonne Universités

${ }^{4}$ National Oceanography Centre, European Way, Southampton, SO14 3ZH, UK

${ }^{5}$ Met Office Hadley Centre, Exeter, UK

${ }^{6}$ National Center for Atmospheric Research, Boulder, USA

Correspondence to: Pablo Ortega (pablo.ortega@bsc.es)

\section{Supplementary Figures}



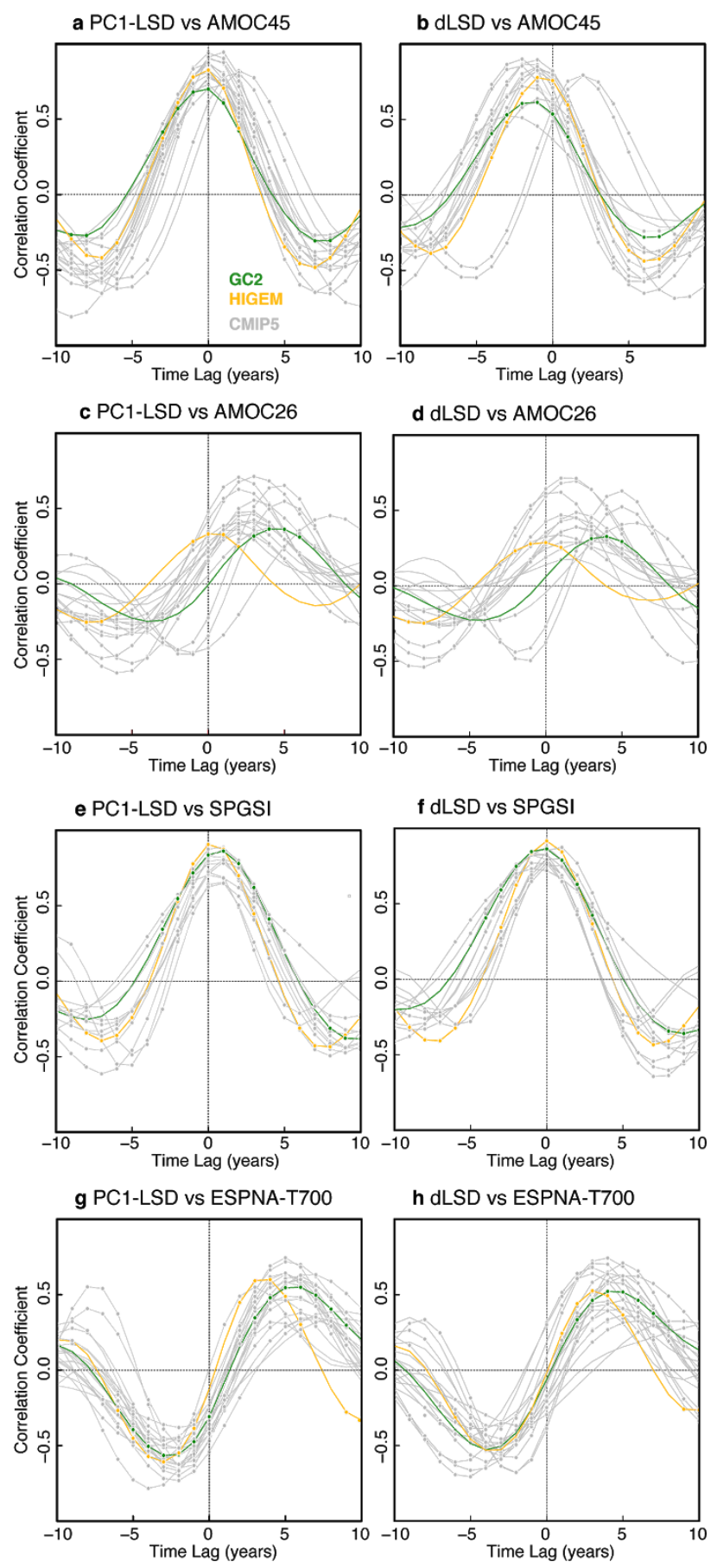

Supplementary Figure 1: a Lead-lag correlations across the picontrol ensemble between PC1-LSD and the maximum AMOC streamfunction at $45^{\circ} \mathrm{N}$ after the Ekman transport is removed (AMOC45). Correlations are based on 10-year running trends. . For positive lags, PC1-LSD leads. Significance is assessed as in Figure 2d and indicated with a circle. b The same as in a but for dLSD index. c-h The same as in $a-b$ but with respect to the AMOC26 (without the Ekman component), the SPGSI and ESPNA-T700 indices, respectively. 


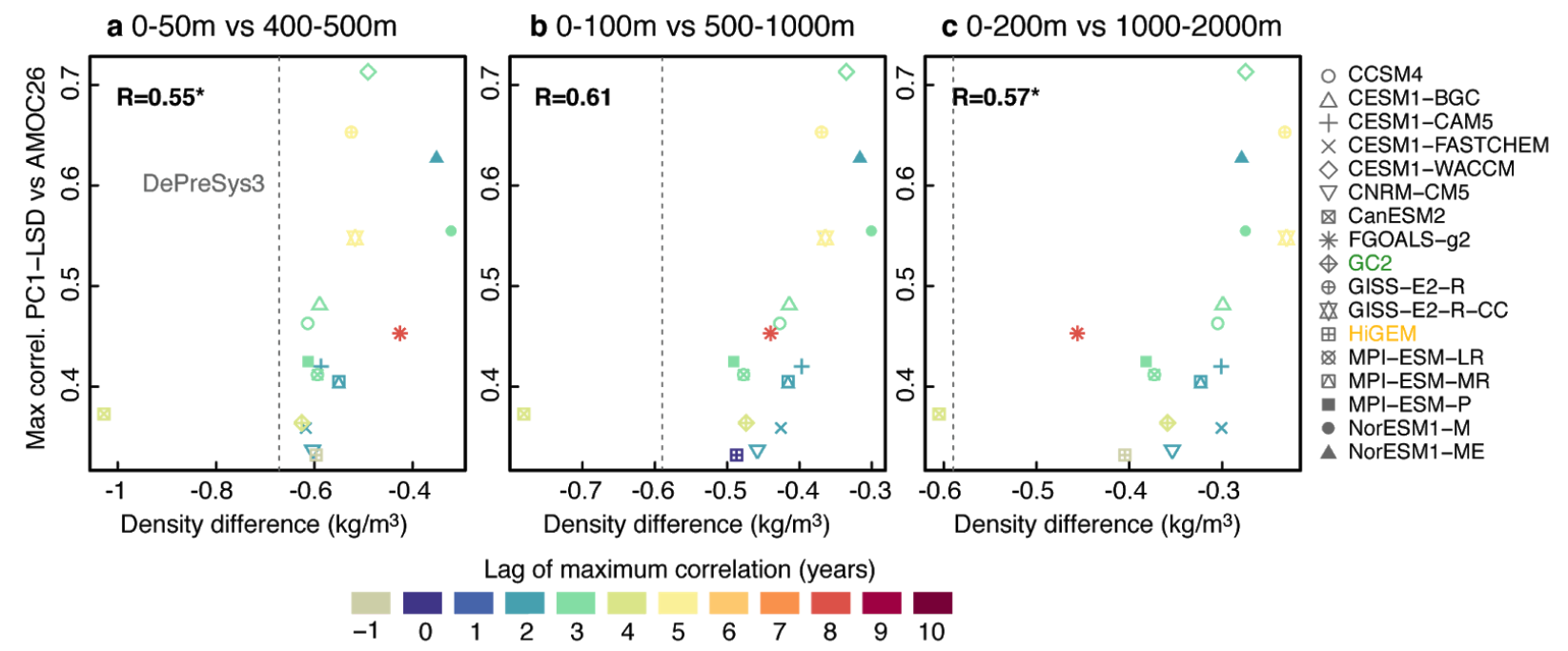

Supplementary Figure 2: a Scatterplot of the maximum correlations at any lag between PC1-LSD and AMOC26N (without the Ekman component) against the climatological mean of the Labrador Sea Density stratification index (computed as the difference of the vertical means in the levels 0-50 m minus the vertical means in the levels 400-500 $\mathrm{m}$; see Fig. 1). The maximum correlations are based on 10-year running trends. The correlation coefficient between the two metrics is shown in the top-left corner. The presence of an asterisk indicates that the correlation is significant at the $95 \%$ confidence level. Colors indicate the lag at which the maximum correlation between PC1-LSD and AMOC26 is obtained. The grey vertical lines depict the mean stratification value in the DePreSys3 assimilation run for the reference period 1960-2013. b-c The same as in $a$ but for stratification indices defined between the levels $0-100 \mathrm{~m}$ vs $500-1000 \mathrm{~m}$ and $0-200 \mathrm{~m}$ vs $1000-2000 \mathrm{~m}$, respectively. 